\title{
Production of a reducing environment for metallurgy using nuclear energy
}

\author{
V. Klimova, V. Pakhaluev \& S. Shcheklein \\ Department of Nuclear Power Plants and Renewables, \\ Ural Federal University, Russia
}

\begin{abstract}
One of the promising ways to reduce the energy consumption of metal production is the use of direct reduction technologies. In this paper the prospects of involving a nuclear energy source based on high temperature gas-cooled nuclear reactors (HTGR) in the production of reducing gases for metallurgy are considered. The description and main thermohydraulic features of a prospective methane converter with radial flow of a gaseous reaction mixture are given. The heat produced in HTGR is fed to the converter through a pipe system with a helium flow. The application of the methane converter with a radial gas flow scheme provides more compact construction and lower hydraulic loss in comparison with the traditional axial scheme.
\end{abstract}

Keywords: high temperature gas-cooled reactor, methane conversion, synthetic gas, reducing environment.

\section{Introduction}

Nowadays, we can observe active development of atomic energy as an electricity source. But the share of electricity in total energy resources' consumption is about $20 \%$. The remaining $80 \%$ of oil, natural gas, coal and other energy resources is expended for housing and transport needs and the energy supply of various industrial technological processes. Developing large-scale nuclear hydrogen energy can supplant expensive hydrocarbonic fuel from the energy sector and save oil and natural gas for the branches of industry where it is more difficult to replace them [1].

The iron and steel industry has the second position after power engineering in the rank of consumers of fuel resources. One of the promising ways to reduce the 
energy consumption of metal production is the use of technologies of direct reduction of metals, especially iron, from the ore. Direct reduced iron (DRI) is produced from oxides by destroying chemical bounds without melting. The result of direct iron reducing is the highly metalized material with a complexity of features (composition, structure, size etc.) which depends on the applied technology. The main DRI consumer is electrometallurgy, but it can also be processed in open-hearth and converter furnaces instead of scrap metal. The blast-furnace process is entirely excluded in this type of production [2].

That is why raw material that is achieved in the direct iron reducing process allows us to reduce the negative impact of metallurgy on the environment, including the reduction of carbon dioxide emission into the atmosphere. The methods of metal production without blast-furnaces and, in the first place, the direct reduction method are widespread all over the world. It is connected first of all with an increasing need for pure metal.

\section{The technology of direct iron reduction with hydrogen-containing gases}

There are two factors that limit the development of direct reduction methods. The first one is the need for rich ore to maximize productivity. The second is the need of natural gas or other reducing gases and a sufficient quantity of energy resources to run the process effectively.

One of the most effective ways to directly reduce iron from the ore is the application of hydrogen-containing gases. The technologies of direct iron reduction from oxides (the so-called DRI and HBI technologies) are used actively worldwide. These methods consume up to $400 \mathrm{~m}^{3}$ of natural gas per 1 ton of metallic pellets produced [2].

Today we can observe the trend of reduction in iron production profitability in the conditions of the rise in prices of the organic fuel.

In the DRI process, the enriched iron ore is reduced by the certain gas mixture under the high temperature conditions. Iron ore is usually processed in the form of pellets that contain about $70 \%$ of iron by weight. The reduction process can be run in shaft furnaces or rotating pipe furnaces in the atmosphere of reducing gases such as carbon monoxide and hydrogen [2].

Let's examine the process of DRI production on the example of the Midrex process. Midrex company is the recognized leader of the DRI facilities market which has worked in this sphere since 1969.

Enriched iron ore in the form of pellets or lumps is charged into the Midrex shaft furnace. The typical raw material for the process has the following content:

- $\mathrm{Fe}-67 \%$

- $\mathrm{SiO}_{2}+\mathrm{Al}_{2} \mathrm{O}_{3}-3 \%$

- $\mathrm{S}-0.008 \%$

- $\mathrm{TiO}_{2}-0.15 \%$

- $\mathrm{P}-0.03 \%$.

The overall reduction reactions are

$$
\mathrm{Fe}_{\mathrm{X}} \mathrm{O}_{\mathrm{Y}}+\mathrm{CO}=\mathrm{Fe}+\mathrm{CO}_{2}
$$




$$
\mathrm{Fe}_{\mathrm{X}} \mathrm{O}_{\mathrm{Y}}+\mathrm{H}_{2}=\mathrm{Fe}+\mathrm{H}_{2} \mathrm{O} \text {. }
$$

The product of the Midrex process contains $90 \ldots 94 \% \mathrm{Fe}$.

The process of iron reducing from oxides runs by sequential transition from higher to lower oxides. It can be schematically described as

- $\mathrm{Fe}_{2} \mathrm{O}_{3} \rightarrow \mathrm{Fe}_{3} \mathrm{O}_{4} \rightarrow \mathrm{FeO} \rightarrow \mathrm{Fe}$; temperature higher than $570^{\circ} \mathrm{C}$

or

- $\mathrm{Fe}_{2} \mathrm{O}_{3} \rightarrow \mathrm{Fe}_{3} \mathrm{O}_{4} \rightarrow \mathrm{Fe}$; temperature lower than $570^{\circ} \mathrm{C}$.

Hydrogen has a lower reducing ability than carbon monoxide if the reaction temperature is lower than $810^{\circ} \mathrm{C}$, but in the temperature range higher than $810^{\circ} \mathrm{C}$ it becomes a much more stronger deoxidizer [2].

The rate of the oxides reducing process increases significantly if pure hydrogen is used as a reducer. Due to technical and economic difficulties of hydrogen production, the sphere of its application is limited and it is used for the production of metallic powders and the creation of nitric and hydrogenous atmosphere for the processes of thermochemical treatment of metals.

The large-scale production of metal pellets is connected with the reducing processes based on synthetic gas (mixture of $\mathrm{CO}$ and $\mathrm{H}_{2}$ ). Synthetic gas can be produced in a steam methane converter or Midrex reformer from natural gas. It also can be obtained from coal or coking gas. The reducing gas composition affects the quantity of the iron produced. The greatest iron output was achieved when the $\mathrm{H}_{2}$ to $\mathrm{CO}$ ratio in the reducing gas fed into the shaft furnace for the reducing process was equal to 1 .

The reaction of steam methane conversion

$$
\begin{aligned}
& \mathrm{CH}_{4}+\mathrm{H}_{2} \mathrm{O}=\mathrm{CO}+3 \mathrm{H}_{2}-206 \mathrm{~kJ} / \mathrm{mol}, \\
& \mathrm{CO}+\mathrm{H}_{2} \mathrm{O}=\mathrm{CO}_{2}+\mathrm{H}_{2}+41 \mathrm{~kJ} / \mathrm{mol}
\end{aligned}
$$

in view of high endothermicity, is run in pipe furnaces with a large amount of upright pipes filled with a dispersed catalyst and heated by gas burners from the outer side. To heat the steam and methane mixture and to supply the heat of endothermic reaction, a large amount of gas is consumed; up to $50 \%$ of the total amount.

The production of hydrogen by electrolysis for the purposes of metallurgy is limited by high energy consumption of the electrolysis process. Electrolysis energy consumption is 5 times higher than the inherent energy capacity of the received hydrogen. The other problem is the high level of $\mathrm{CO}_{2}$ emission due to the contribution of thermal power plants producing electricity.

The production of hydrogen and hydrogen containing gases in the process of methane conversion has better efficiency and ecological features than electrolysis. For example, the method energy consumption exceeds hydrogen energy capacity by 1.14 times.

\section{Application of HTGR heat for the production of hydrogen containing gases}

Economically efficient large-scale hydrogen and hydrogen containing gases production can be provided by the use of high temperature gas-cooled nuclear reactor (HTGR) heat. 
A nuclear facility for energotechnological purposes consists of a reactor circulation circuit which includes HTGR with helium cooling, gas blowers and other systems, and a technological circuit including chemical reactors. To ensure the facility safety and reliability, nuclear and technological circuits are separated by an intermediate helium to helium heat exchanger. The nuclear reactor helium outlet temperature is $950 \ldots 1000^{\circ} \mathrm{C}$. The intermediate heat exchanger helium outlet temperature is $900 \ldots 950^{\circ} \mathrm{C}$, which is sufficient for the steam methane conversion reaction [3].

A schematic diagram of the facility is shown in fig. 1.

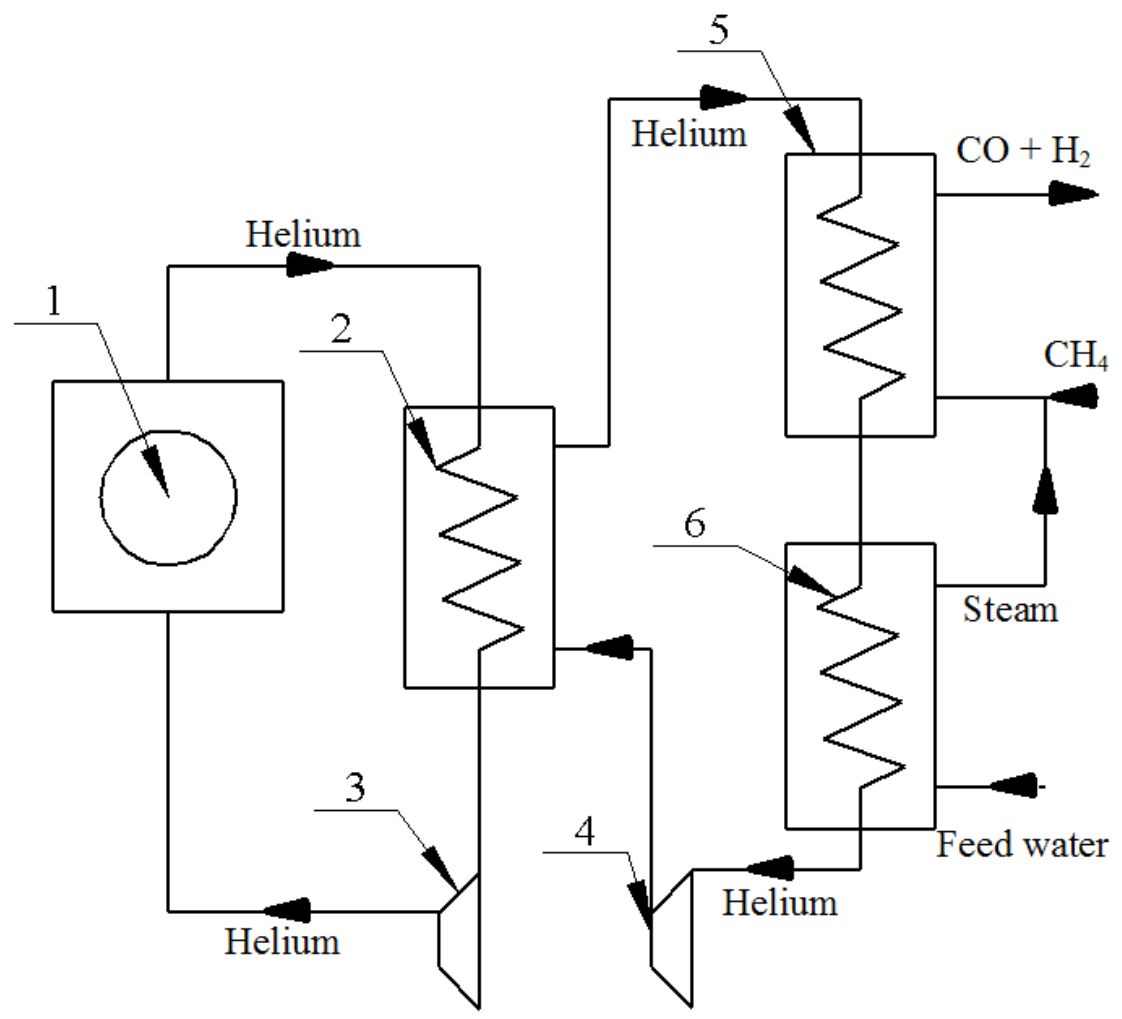

Figure 1: Energotechnological facility schematic diagram.

Helium of the first circuit heated in HTGR (1) is fed into an intermediate heat exchanger (2), where it transfers heat to the helium of the second circuit. The helium of the second circuit is directed into the pipes of the methane converter (5) where it supplies the endothermic reaction with heat. After the converter, helium with lower temperature arrives in the steam generator (6) to produce steam. Helium circulation is provided by gas blowers (3) and (4).

It is possible to create an energotechnological complex that consists of a HTGR reactor and a methane conversion plant on the base of nuclear reactor. 
Synthetic gas produced at the plant can be transported through pipelines to the consumers which are represented not only by the metal industry but also ammonia, methanol and synthetic fuel producers. It is expedient to provide the methane converter heating through a system of pipes with the helium of the second circuit circulating inside. The catalyst granules fill the space between tubes of the converter. The reacting mixture of gases can flow in an axial or radial direction relative to the apparatus axis.

Apparatuses with radial gas flow in the granular layer have substantial advantage in comparison with axial flow apparatuses because of significantly lower flow pressure loss in the layer. This leads not only to the productive capacity rise and energy saving but also to the reduction of specific quantity of metal due to better apparatus compactness [3].

To intensify the heat transfer process in the tube furnaces with a catalyst layer, the multipass circulation of reacting gases is usually applied. In this case a convergent-divergent regime is realized in radial flow apparatuses. The reacting gas flow is accelerated in one section and is decelerated in the following section.

The schematic diagram of a methane converter with radial flow is shown in fig. 2. The catalyst granules are laid in the cavity between two coaxial cylindrical collectors of greater and lesser radius with walls perforated for the gas passage.

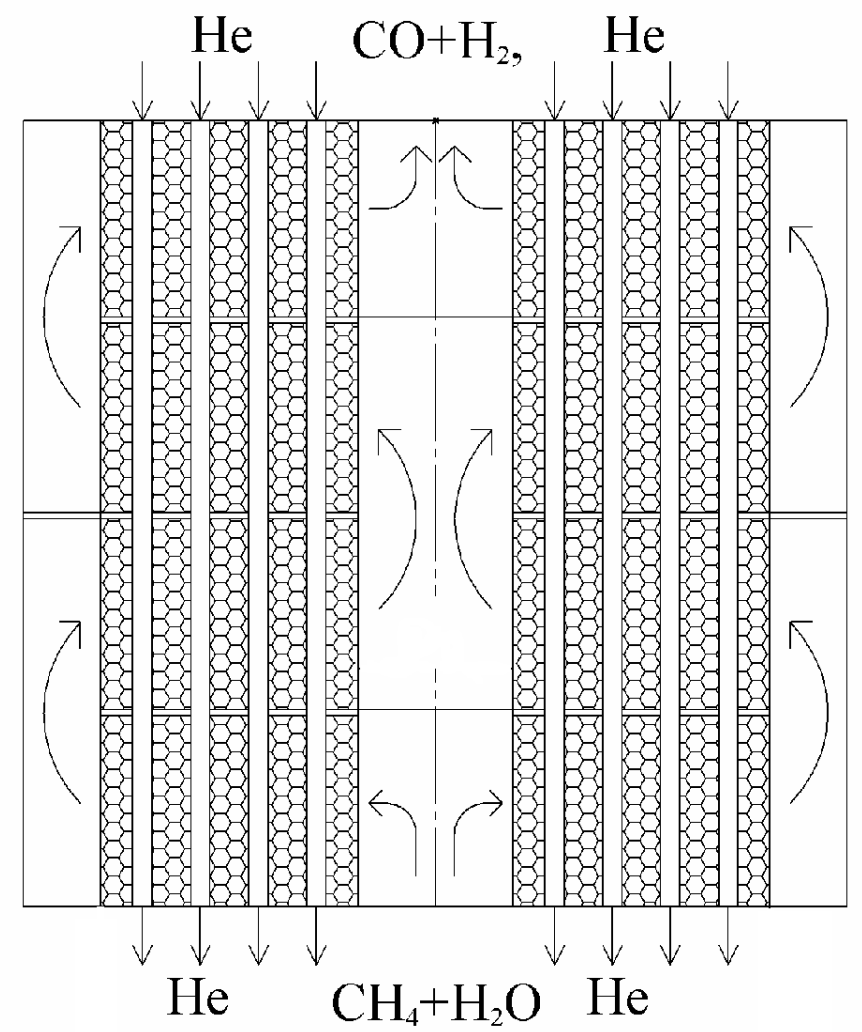

Figure 2: Steam methane converter schematic diagram. 
For design calculation, it is necessary to consider the peculiarities of the radial gas flow connected with the flow velocity variation from the entrance of the distributing collector to the exit of the receiving collector [3].

The heating of steam and methane mixture $\left(\mathrm{CH}_{4}+\mathrm{H}_{2} \mathrm{O}\right)$ is implemented by a system of vertical pipes with a helium heat transfer agent. The intensity of heat transfer from the helium inside the pipes to the reacting mixture can be sufficiently increased with the use of different methods of flow swirling inside the pipes and rational choice of radial flow mode.

The calculation of main dimensions of the steam methane converter with radial gas $\left(\mathrm{CH}_{4}+\mathrm{H}_{2} \mathrm{O}\right)$ flow was made with the use of experimental formula given in $[3,4]$. An experimental facility with granular layer filling the space between tubes was used to achieve data on the heat exchange between the gas inside the pipes and the gas moving in the granular layer.

The results of thermohydraulic calculation are given in tab. 1 .

Table 1: The results of thermohydraulic calculation of the methane converter.

\begin{tabular}{|c|c|c|c|}
\hline \multirow{2}{*}{ Parameter } & \multicolumn{3}{|c|}{ Methane rate (tons per hour) } \\
\hline & 500 & 1000 & 1500 \\
\hline Quantity of heat, MW & & & \\
\hline total & 6.85 & 13.71 & 20.56 \\
\hline for gas mixture heating & 0.93 & 1.87 & 2.80 \\
\hline for endothermic reaction & 5.92 & 11.84 & 17.76 \\
\hline Helium rate, $\mathrm{kg} / \mathrm{s}$ & 17.6 & 35.2 & 52.8 \\
\hline $\begin{array}{l}\text { Characteristics of heat exchange tubes } \\
\text { length, } \mathrm{mm} \\
\text { diameter } \mathrm{x} \text { wall thickness, } \mathrm{mm}^{2} \\
\text { spacing, } \mathrm{mm}\end{array}$ & \multicolumn{3}{|c|}{$\begin{array}{l}3000 \\
40 \times 2.5 \\
56\end{array}$} \\
\hline $\begin{array}{l}\text { Heat transfer coefficient, } \mathrm{W}\left(\mathrm{m}^{2} \cdot \mathrm{K}\right) \\
\text { for heating zone } \\
\text { for reaction zone }\end{array}$ & $\begin{array}{l}120.6 \\
155.5\end{array}$ & $\begin{array}{l}133.8 \\
168.8\end{array}$ & $\begin{array}{l}143.4 \\
178.4\end{array}$ \\
\hline Heat exchange surface, $\mathrm{m}^{2}$ & 231.9 & 425.5 & 601.9 \\
\hline Converter diameter, $\mathrm{m}$ & 1.62 & 2.10 & 2.45 \\
\hline Pressure loss for the reacting gas, $\mathrm{kPa}$ & 3.79 & 12.5 & 24.6 \\
\hline Pressure loss for helium, $\mathrm{kPa}$ & 3.17 & 3.71 & 4.14 \\
\hline Needed catalyst volume, $\mathrm{m}^{3}$ & 0.24 & 0.48 & 0.72 \\
\hline Space between tubes, $\mathrm{m}^{3}$ & 3.2 & 5.5 & 7.5 \\
\hline
\end{tabular}

\section{Conclusion}

In the nearest future, reducing gases for metallurgy will be produced with the use of organic raw material and a steam methane conversion process. Creation of energotechnological complexes on the base of HTGR allows us to reduce the organic fuel consumption and to stimulate the development of hydrogen 
production technologies based on thermochemical and combined thermoelectrical cycles.

\section{References}

[1] Ponomarev-Stepnoy, N.N., Stolyarevskii, A.Ya. \& Pakhomov, V.P. Nuclear-Hydrogen Power Engineering, Energoizdat: Moscow, pp. 76-89, 2008.

[2] Yaroshenko, Yu.G., Gordon, Ya.M. \& Hodorovskaya, I. Yu, Energy Efficient and Resource-Saving Technologies in Iron-and-Steel Industry, UIPC: Ekaterinburg, pp. 526-576, 2012.

[3] Klimova, V.A. \& Pakhaluev, V.M. Energotechnological system of longdistance atomic heat supply with radial gas flow installations. International Scientific Journal for Alternative Energy and Ecology, 3(107), pp. 26-30, 2012.

[4] Klimova, V.A., Pakhaluev, V.M. \& Shcheklein, S.E. Numerical simulation and experimental investigations of hydrodynamics and heat transfer for radial gas flow in an apparatus with ball packing. Thermal engineering, 4, V.58, pp. 325-330, 2011. 